\title{
Electronic Structure Calculations of Vacancies and Their Influence on Materials Properties
}

\author{
P.A. Sterne \\ J. van Ek \\ R.H. Howell \\ This paper was prepared for submittal to the \\ International Conference on Advanced Materials Spring Meeting \\ Strasbourg, France \\ June 16-20, 1997
}

August 1997

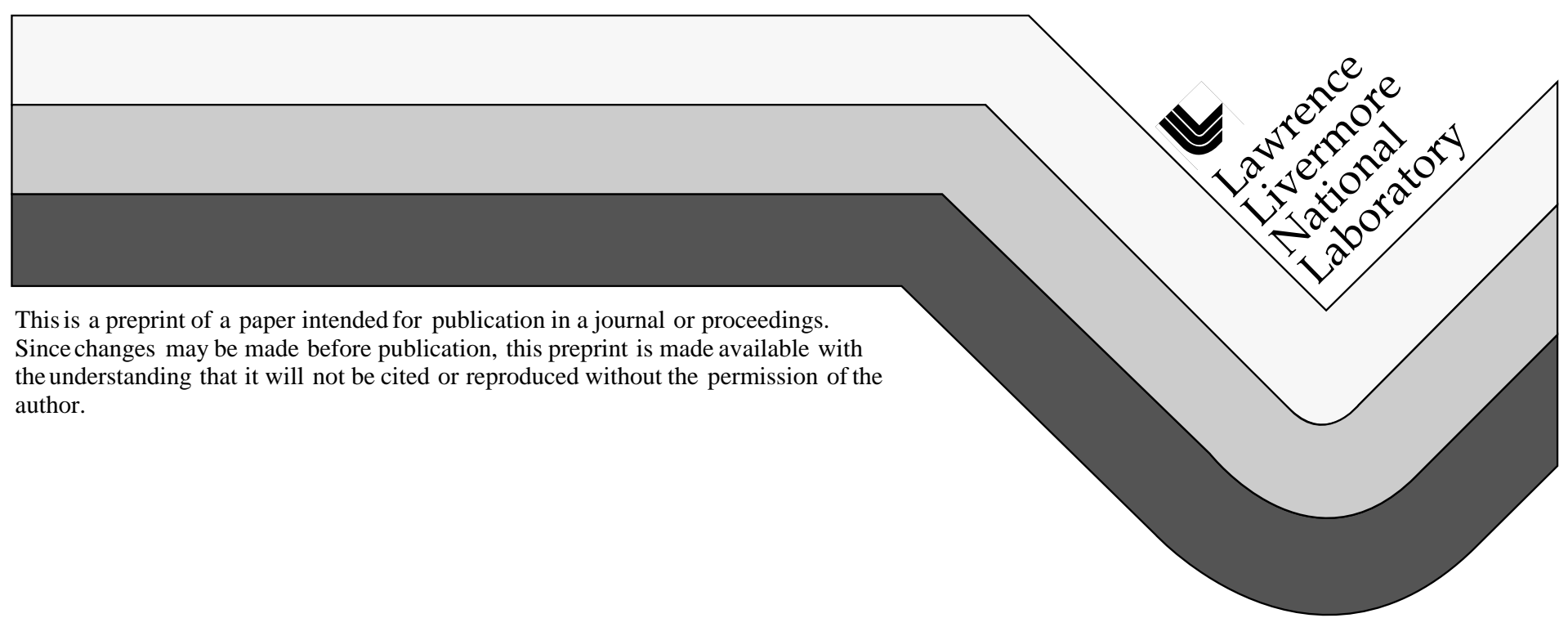




\section{DISCLAIMER}

This document was prepared as an account of work sponsored by an agency of the United States Government. Neither the United States Government nor the University of California nor any of their employees, makes any warranty, express or implied, or assumes any legal liability or responsibility for the accuracy, completeness, or usefulness of any information, apparatus, product, or process

disclosed, or represents that its use would not infringe privately owned rights. Reference herein to any specific commercial product, process, or service by trade name, trademark, manufacturer, or otherwise, does not necessarily constitute or imply its endorsement, recommendation, or favoring by the United States Government or the University of California. The views and opinions of authors expressed herein do not necessarily state or reflect those of the United States Government or the University of California, and shall not be used for advertising or product endorsement purposes. 


\title{
Electronic structure calculations of vacancies and their influence on materials properties
}

\author{
P.A. Sterne \\ Department of Physics, University of California, Davis, CA 95616, USA \\ and \\ Lawrence Livermore National Laboratory, P.O. Box 808, L-407, Livermore, CA 94550, USA \\ J. van Ek \\ Department of Physics, Tulane University, New Orleans, LA 70118, USA

\section{R.H. Howell} \\ Lawrence Livermore National Laboratory, P.O. Box 808, L-280, Livermore, CA 94550, USA
}

\begin{abstract}
We provide two examples to illustrate how electronic structure calculations contribute to our understanding of vacancies and their role in determining materials properties. Diffusion and electromigration in aluminium are known to depend strongly on vacancies. Electronic structure calculations show that the vacancy-impurity interaction oscillates with distance, and this leads to an explanation for both the increased electromigration resistance and the slow impurity diffusion for copper in aluminium. Calculations of vacancies in plutonium have been used in conjunction with positron annihilation lifetime measurements to identify the presence of helium-filled vacancies. Helium stabilization of vacancies can provide the precursor for subsequent vacancy-related changes in materials properties.
\end{abstract}

Proceedings of Symposium D, ICAM/EMRS '97 Meeting, Strasbourg, France, June 16-20, 1997.

To be published in Computational Materials Science 


\section{INTRODUCTION}

Vacancies contribute in a number of ways to the properties of materials. Their effects and interactions must be taken into account in describing a variety of processes and materials aging issues since they are intrinsic defects and their influence cannot be eliminated at finite temperatures. Vacancies play a crucial role in diffusion, the random motion of atoms in a solid, and in electromigration, the transport of atoms under the influence of electric fields and currents. Vacancies can also contribute significantly to resistivities in metals at elevated temperatures. Radiation damage in crystalline materials often produces Frenkel defects, i.e. vacancy-interstitial pairs, and although the vast majority of these recombine rapidly, vacancies can still lead to changes in microstructure with subsequent changes in materials properties.

In this paper we consider the role played by vacancies in two different materials problems. The first example concerns the role of vacancies in diffusion and electromigration in dilute alloys, with particular attention to the case of $\mathrm{Cu}$ in $\mathrm{Al}$. The variation of the impurity-vacancy interaction with distance is shown to be significant. The second example addresses the detection by positron annihilation of self-irradiation-induced vacancies in plutonium using positron annihilation. Theoretical calculations of positron lifetimes based on first principles electronic structure methods play an essential role in interpreting the experimental data in this case.

\section{DIFFUSION AND ELECTROMIGRATION}

Bulk diffusion of substitutional impurities and self-diffusion in close packed fcc and hcp metals are mediated by vacancies $[1,2]$. Only the atoms adjacent to vacancies can change their lattice position at any given moment, so diffusion is strongly dependent on the vacancy formation energy which controls the number of thermal vacancies present in the system. Diffusion also depends on the migration energy, the amount of energy required to carry an atom over the energy barrier from its original lattice position into the vacancy site. Self-diffusion is generally described in terms of an activation energy $Q_{0}$ which combines these terms:

$$
D=D_{0} e^{-Q_{0} / k T}
$$


The diffusion of substitutional impurities can also be represented in this exponential form, but with a different activation energy [1, 2, 3]:

$$
D_{\text {imp }}=D_{2} e^{-Q_{2} / k T}
$$

It is customary to define the activation energy difference:

$$
\Delta Q=Q_{2}-Q_{0}=\Delta E_{h}+E_{i-v}-C,
$$

where $\Delta E_{h}$ is the difference in migration energies between bulk and impurity atoms, $E_{i-v}$ is the impurity-vacancy interaction energy, and $C$ is a correlation term which is typically small and has a slight temperature dependence.

The impurity-vacancy interaction enters into the activation energy difference in a very direct way $[3,4]$ - impurities with negative impurity-vacancy interaction energies attract vacancies to nearest-neighbour sites thereby lowering the activation energy, while positive impurity-vacancy interactions indicate repulsion, and an increase in the activation energy. If we assume that the migration energies are similar to those for the bulk atoms, then impurities with attractive impurityvacancy interactions will tend to have lower activation energies than self-diffusion, and will therefore be fast diffusers. In contrast, those that repel vacancies will have higher activation energies, leading to slower diffusion rates than those observed for self-diffusion. Table I shows some fast and slow diffusers in $\mathrm{Al}$, with corresponding values of $\Delta Q[5,6]$. Note that $\mathrm{Cu}$ is a slow diffuser in $\mathrm{Al}$, suggesting that $\mathrm{Cu}$ repels vacancies from its nearest-neighbour shell.

The kinetic five-frequency model is widely used to describe diffusion in fcc lattices [3]. In this model, frequencies are assigned to the various jumps that atoms can make into adjoining vacancy sites. The individual frequencies depend on the activation energies for individual atomic processes, so the impurity-vacancy interaction enters indirectly through the values of the frequencies for jumps that change the impurity-vacancy separation. The model effectively includes only nearest-neighbor impurity-vacancy interactions since it distinguishes only atomic jumps that involve impurities and vacancies in neighboring sites either before or after the jump is completed. All other atomic jumps are represented in terms of a single "bulk" frequency, regardless of impurityvacancy separation. 
In cases where there is a difference in charge between the impurity and the host, the impurityvacancy interaction has been estimated on the basis of electrostatic screening $[1,7,8]$,

$$
E_{i-v}(r) \sim \frac{e^{-q r}}{r}
$$

where $\mathrm{q}$ is the inverse screening length. This interaction is generally evaluated and used only at the nearest-neighbour distance. However, if it is taken to further distances, it clearly represents a monotonic variation of the impurity-vacancy interaction with distance. First principles electronic structure calculations can assess the applicability of these models to dilute alloys. By examining the impurity-vacancy interaction as a function of distance, it is possible to determine quantitatively if the nearest-neighbour term alone is sufficient for an accurate description of the impurity-vacancy interaction in diffusion, or if further neighbour terms should be included. It is also possible to assess the reliability of simple models like the statically screened electrostatic model for describing the interactions at longer distances, and if the interactions are indeed monotonic in nature.

Vacancies also play an important role in electromigration in which the random atomic diffusion is biased by an applied electric field leading to net mass transport in one direction. Electromigration in aluminium interconnects is one of the major failure mechanisms for microelectronic devices [9]. It is well known that the addition of a few atomic percent of copper in aluminium leads to a significant increase in electromigration resistance, improving the operating lifetime of the microelectronic devices. The mechanism by which $\mathrm{Cu}$ increases electromigration resistance is unknown, however. There have been several suggestions, many of which are based on an assumed attractive interaction between the vacancies and the impurity $\mathrm{Cu}$ atom $[10,11,12]$. These models appear to be in contradiction with diffusion data since a repulsive impurity-vacancy interaction is required to account for the slow diffusion[5] of $\mathrm{Cu}$ in $\mathrm{Al}$. This apparent contradiction has stimulated the present study of impurity-vacancy interactions in Al.

We have used a first principles self-consistent electronic structure approach to calculate the total energy as a function of impurity-vacancy separation for a variety of substitutional impurities in aluminium. The linear muffin tin orbital method together with the atomic sphere approximation [13] and the local density approximation [14] were used in a supercell geometry with 32 atoms, 
including an impurity atom and an empty sphere on the vacancy site. The all-electron calculations included fully relativistic core states and scalar-relativistic valence states. The basis set included $s, p$ and $d$ orbitals on all sites, and the Brillouin zone integration was performed with a mesh of 125 k-points. Convergence in k-point sampling was checked by performing additional calculations with 216 and $343 \mathrm{k}$-points. The most significant approximation in this approach is the neglect of lattice relaxation around the vacancy and impurity sites. The implications of this approximation for our results will be addressed below.

The calculations were performed with the impurity atom at nearest-neighbour, second-neighbour, third-neighbour and fourth-neighbour positions with respect to the vacancy in the same unit cell. The total energies were converged to $1 \mathrm{meV}$ or better in all cases. Seven different impurity atoms were considered to ensure reliability in the observed trends - $\mathrm{Sc}, \mathrm{Ti}, \mathrm{Cu}, \mathrm{Mg}, \mathrm{Si}, \mathrm{Ag}$ and $\mathrm{Au}$. We observe a pronounced oscillation in the total energy with impurity-vacancy separation for all seven impurities. The amplitude of this oscillation is numerically significant, and is unaffected when we increase the number of k-points, indicating that it is not an artefact of the calculation.

The oscillatory form of the total energy suggests that the impurity-vacancy interaction itself may oscillate with distance. The well-known Friedel oscillations [15] in the charge density around an impurity provide a natural source of an oscillating interaction in dilute alloys. The Friedel oscillations arise due to the metallic screening of the impurity and vacancy potentials by the host electrons, which in the case of Al have a significant free-electron character.

In order to check if our results are consistent with a Friedel-like mechanism, we have fitted our total energies to an oscillatory form [15]. We assume that the interaction between any given vacancy and impurity can be written as

$$
\varepsilon\left(R_{i}\right)=A \frac{\cos \left(B R_{i}+\phi\right)}{R_{i}^{3}},
$$

where $R_{i}$ is the distance to an impurity in the $i$-th shell around the vacancy and $A, B$ and $\phi$ are determined from the fitting procedure. This functional form should be valid for distances longer than screening length, which is less than the nearest-neighbour distance in Al. The calculated total energies include multiple impurity-vacancy interactions due to the supercell used in the calcula- 
tions, so the fitting procedure must take this into account in determining the parameters $A, B$ and $\phi$. We write the total energy for a given configuration as

$$
E_{i}=C+\sum_{j} n_{i j} \varepsilon\left(R_{j}\right)
$$

where the total energies $E_{i}$ for the configuration with the impurity in shell $i$ around the vacancy is put equal to the sum over all the impurity-vacancy terms together with a constant background energy $C$. The constant $C$ is eliminated analytically, and the fitting procedure is performed using a standard non-linear fitting procedure [16] to yield the parameters $A, B$ and $\phi$. If the oscillations truly arise from a Friedel-like form, the value of $B$ will be close to twice the Fermi wavevector for $\mathrm{Al}, 2 k_{F}=1.85 a_{0}^{-1}$

Table 2 shows that the values of $B$ resulting from the fit are close to twice the free-electron Fermi wavevector in $\mathrm{Al}$ for all seven impurities considered here, as expected for a Friedel-based mechanism. In fact, the values of $B$ fall slightly below the free-electron estimate, which may be due to an effective reduction in $k_{F}$ due to Umklapp processes in the crystal. Nevertheless, they are all within 4-16\% of the free-electron value, which strongly suggests that the observed oscillations in total energy originate from a Friedel-like mechanism.

Figure 1 shows the impurity-vacancy interaction energy as a function of separation based on the fitted values obtained from the total energy calculations. The positions of the various atomic shells around the vacancy are indicated. All of the curves show a pronounced oscillatory form, although the amplitude is small in the case of $\mathrm{Mg}$. There is clearly a strong repulsion at the nearestneighbour site for $\mathrm{Sc}, \mathrm{Cu}$ and $\mathrm{Ti}$, indicating that they are likely to be slow diffusers in $\mathrm{Al}$. This is consistent with experiment, which shows that both $\mathrm{Cu}$ and $\mathrm{Sc}$ are slow diffusers. No experimental data is available for $\mathrm{Ti}$, so this constitutes a theoretical prediction that $\mathrm{Ti}$ will be a slow diffuser in Al. The Si and Au impurities show an attractive impurity-vacancy interaction at the nearestneighbour site, and this is consistent with the observation that they are fast diffusers in $\mathrm{Al} . \mathrm{Mg}$ and Ag show small repulsive impurity-vacancy nearest-neighbour interactions, so the contribution to the diffusion activation energy from the migration energy may be significant for these impurities. In addition if we allow for lattice relaxation, the impurity-vacancy distance will decrease and the 
curves in figure 1 suggest that this will further reduce the impurity-vacancy interaction energy for $\mathrm{Ag}$ and $\mathrm{Mg}$. We note that this effect will also lower the nearest-neighbour interaction for $\mathrm{Au}$ which in the absence of relaxation is slightly more attractive at the second-neighbour site than the nearest-neighbour site. The impurities $\mathrm{Mg}, \mathrm{Si}, \mathrm{Ag}$ and $\mathrm{Au}$ therefore all have attractive or only slightly repulsive impurity-vacancy interactions at the nearest-neighbour site, consistent with their observed behaviour as fast diffusers in $\mathrm{Al}$, while the much larger impurity-vacancy repulsions at the nearest-neighbour site for $\mathrm{Sc}$ and $\mathrm{Cu}$ are consistent with their observed slow diffusion.

Table 2 also shows the difference in energy between the nearest-neighbour and second-neighbour impurity-vacancy configurations. These numbers are of the same order as published dissociation and binding energies $[3,4,17,18,19]$, and in good quantitative agreement with first principles calculations for nearest- and second-neighbour impurity-vacancy interaction energies for $3 d$ impurities in $\mathrm{Al}$ [20]. Note that for the slow diffusers, it is favorable for the atom to sit at the secondneighbour position from the vacancy site. In fact, this is an attractive interaction at this distance the second-neighbour site is more favourable than infinite separation, so that at a sufficiently low temperature the vacancy is bound to the impurity at the second-neighbour site.

The calculations confirm the validity of an oscillating Friedel-like form for the vacancy-impurity interaction, and clearly indicate that models relying on nearest-neighbour interactions alone are not sufficient to describe the impurity-vacancy interaction for all substitutional impurities. The traditional application of the kinetic model based only on nearest-neighbour interactions is very reasonable for impurities with attractive impurity-vacancy interactions, such as $\mathrm{Si}$ in $\mathrm{Al}$ and electropositive impurities in noble metals [1], where the simple screened-Coulomb electrostatic theory works well. However for systems with repulsive impurity-vacancy interactions, the oscillatory nature of the interaction, which has its origin in the very general Friedel oscillations, results in the possibility of an attractive impurity-vacancy interaction at the second-neighbour or some further neighbour site. This attraction, which can significantly affect the diffusion, is completely neglected in the widely used five-frequency kinetic model.

We noted in the introduction that $\mathrm{Cu}$ in $\mathrm{Al}$ is a slow diffuser, implying a repulsive impurityvacancy interaction. At the same time, the increase in $\mathrm{Al}$ electromigration resistance with $\mathrm{Cu}$ 
doping implies that the $\mathrm{Cu}$ impurity attracts vacancies, thereby inhibiting their participation in electromigration. This apparent contradiction is resolved by our results, which show that the impurity-vacancy interaction is repulsive at the nearest-neighbour site, but that there is a significant attraction at the second-neighbour site. While this attraction is not sufficient to permanently bind the vacancy to the $\mathrm{Cu}$ site, it may retard vacancy motion sufficiently to result in an increased electromigration resistance.

Lattice deformation around defects can significantly affect the total energies obtained from the electronic structure calculations. The relaxation energy for a vacancy in $\mathrm{Al}$ is in the range $0.1-0.2$ $\mathrm{eV}[21,22]$. This is comparable to the impurity-vacancy repulsion we calculate, so neglecting such a large energy contribution is clearly a source of concern. However, the relaxation around the vacancy will be similar in all configurations, and relatively independent of the impurity-vacancy separation. The impurity-vacancy interaction is only affected by differences in relaxation energies due to different configurations, and not by the size of the relaxation energy itself. For this reason, we have confidence that our results are not adversely affected by neglecting these relaxation energies. Model calculations indicate that relaxation energies vary by only about $0.015 \mathrm{eV}$ depending on the impurity-vacancy separation [21]. This is much smaller than the dissociation energy for an impurity-vacancy pair for $\mathrm{Sc}, \mathrm{Ti}, \mathrm{Cu}$, and $\mathrm{Au}$ (Table 2). The effects of relaxation will be more important in cases where the amplitude $A$ is small, such as $\mathrm{Mg}$ and $\mathrm{Ag}$, and should then be included directly in the total energy calculations. In most other cases, however, relaxation will only have a small quantitative effect on the results. For this reason, our omission of relaxation is not as serious an approximation as it might at first appear since, to first order, the different impurity-vacancy configurations have the same relaxation energy. In addition, the oscillatory nature of the impurityvacancy interaction will not be affected significantly by lattice relaxation since it arises from the Friedel oscillations, a fundamental property of the electronic screening in $\mathrm{Al}$.

The widely used five-frequency kinetic model $[1,2,3]$ for diffusion is convenient since it provides a tractable model that facilitates comparison with experiment. In common with essentially all the analytic work on diffusion on fcc metals, it assumes that only the nearest-neighbour impurityvacancy interaction energy is significant. We have shown here that for some impurities there 
may be a very strong nearest-neighbour repulsion together with a significant second-neighbour attraction. This can modify the diffusion characteristics of the impurity in a way that is not well described in the standard five-frequency model, and clearly calls for the development of a new model to account for the second-shell interactions. The calculated nearest-neighbour repulsion and second-neighbour attraction can also lend support to models of electromigration for $\mathrm{Cu}$ in $\mathrm{Al}$ which rely on an impurity-vacancy attraction to increase electromigration resistance.

\section{VACANCIES IN PLUTONIUM STUDIED BY POSITRON ANNIHILATION}

Radioactive decay in plutonium results in the creation of a large number of vacancies, interstitials, and other defects. An understanding of the behaviour of these defects is important for predicting and understanding how materials properties evolve over time. Each radioactive decay creates a few thousand Frenkel pairs, but the majority of these recombine rapidly, leaving a smaller fraction of uncompensated vacancies and interstitials that can diffuse and interact with other point and extended defects in the lattice.

Positron annihilation lifetime spectroscopy provides a sensitive probe of vacancies and openvolume defects in materials [23]. Positrons annihilate with electrons in the material with a lifetime that depends on the electron charge density in the vicinity of the positron. For defect-free crystals, the positron enters a Bloch-like extended state located primarily in the interstitial region where the repulsion with the similarly charged atomic nuclei is minimized. The positron then annihilates with a characteristic bulk lifetime determined by the electron charge density in this region. In materials with open-volume defects, the positron is naturally attracted to vacancies and voids, again due to repulsion with the atomic nuclei. The lifetime then differs from the bulk lifetime due to the different electron charge density in the vacancy. Vacancies and open volume defects typically have longer lifetimes than the defect-free material, since there are fewer electrons available for annihilation with the positron.

Theoretical calculations of positron lifetimes play a crucial role in interpreting experimental data [24]. Positron lifetimes reflect the local charge density around the positron, with a unique lifetime corresponding to each different positron state. When the experimental data provides a 
single lifetime component, indicating that the positrons all annihilate in a similar state, additional information is required to determine if the observed lifetime value corresponds to a bulk (defectfree) lifetime or a defect. In systems where several different defects may trap positrons, each with their own characteristic lifetime, it is often difficult to match the observed lifetime value with the appropriate defect.

Theoretical calculations of positron lifetimes are based on first-principles electronic structure methods $[24,25,26]$ The electron-electron and electron-positron interactions are all treated within the local density approximation, as is the enhancement of the positron annihilation rate due to the mutual attraction of the electron and positron. The parameters for these interactions are obtained from many-body calculations $[27,28]$ and are independent of measured positron lifetimes, so the method truly constitutes a parameter-free first-principles approach for calculating positron lifetimes. These lifetime calculations have been very successful in reproducing well established bulk lifetimes for many elemental metals [25, 26, 29], as well as vacancy lifetimes in metals, semiconductors [26] and oxides [30].

Positron lifetime measurements on aged plutonium samples produced a single component lifetime of 184 picoseconds (ps) [31], indicating that all the positrons annihilate from the same type of state; either all the positrons remained in extended bulk-states, or they all trapped in similar defect states. Positron lifetime calculations were performed using our first principles approach [25] based on the linear muffin tin orbital method. The resulting bulk lifetime was 139 ps, significantly lower than the measured lifetime. Supercell calculations for a monovacancy produced a converged positron lifetime of around 250 ps with a 64 atom supercell. This is significantly longer than the measured lifetime, indicating that bare monovacancies are not responsible for the observed lifetime. Divacancies and larger vacancy clusters and voids will have even longer lifetimes, corresponding to their lower electron charge densities compared to the monovacancy. The observed lifetime is therefore not consistent with a defect-free lifetime, or an empty vacancy, vacancy clusters, or larger voids.

Helium atoms are produced in plutonium due to alpha decay. There is a strong repulsion between helium atoms and metal atoms due to the closed He $s$-shell of electrons, so helium atoms 
preferentially occupy vacant sites in the lattice if these are available. We have calculated the positron lifetime in a $\mathrm{Pu}$ vacancy containing a $\mathrm{He}$ atom and find a lifetime value in the range 170-195 ps, depending on the position of the He atom. This is in excellent agreement with the measured lifetime value of $184 \mathrm{ps,} \mathrm{leading} \mathrm{to} \mathrm{the} \mathrm{conclusion} \mathrm{that} \mathrm{He-filled} \mathrm{vacancies} \mathrm{are} \mathrm{present} \mathrm{in}$ significant numbers in this aged plutonium sample. Vacancy concentrations of a part per $10^{3}-10^{4}$ are generally sufficient to trap all the positrons into vacancies, so the observation puts a lower limit of one part per $10^{4}$ on the number of helium-filled vacancies in this aged sample.

Helium is known to have an important influence on radiation-induced swelling and subsequent embrittlement in other materials [32]. Helium atoms can stabilize vacancies and small voids and lower the critical radius for cavity growth [33]. Helium stabilization of vacancies is therefore an important precursor for subsequent changes in materials properties, and the identification and observed evolution of these vacancies provides important information for subsequent modeling of aging mechanisms in irradiated materials.

\section{CONCLUSIONS}

We have used the sophisticated apparatus provided by electronic structure calculations to examine key issues associated with realistic materials problems. Calculations of the impurity-vacancy interactions in dilute aluminium alloys provide good agreement with observed trends in diffusion, and also indicate that attractive impurity-vacancy interactions at the second-neighbour lattice distance may be important for understanding diffusion and electromigration properties. Firstprinciples-based positron lifetime calculations indicate that aged plutonium samples contain an appreciable concentration of helium-filled vacancies. In other materials like Al, similar observations have been important in understanding subsequent changes in materials properties due to aging.

\section{ACKNOWLEDGMENTS}

This work was performed under the auspices of the U.S. Department of Energy by Lawrence 
Livermore National Laboratory under contract number W-7405-ENG-48. The authors gratefully acknowledge the Louisiana Board of Regents. 


\section{REFERENCES}

[1] R.J. Borg and G.J. Dienes, An Introduction to Solid State Diffusion (Academic Press, San Diego, 1988).

[2] A.R. Allnatt and A.B. Lidiard, Atomic Transport in Solids (Cambridge University Press, Cambridge, 1993).

[3] A.D. Le Claire, J. Nuc. Mater. 69 \& 70, 70 (1978) and references therein.

[4] M. Doyama, J. Nuc. Mater. 69 \& 70, 350 (1978) and references therein.

[5] Landolt-Börnstein New Series III/26 (Springer, Berlin, 1990).

[6] S. Fujikawa, Sci. Eng. Light Metals RASELM-91 Tokyo, p. 959 (1991).

[7] D, Lazarus, Phys. Rev. 93, 973 (1954).

[8] A.D. Le Claire, Phil. Mag. 7, 141 (1962).

[9] Electromigration \& Electronic Device Degradation, edited by A. Christou, (Wiley \& Sons, New York, 1994).

[10] R. Rosenberg, J. Vac. Sci. Tech. 9, 263 (1971).

[11] P.S. Ho, Phys. Rev. B 8, 4534 (1973).

[12] C. Kim and J.W. Morris, J. Appl. Phys. 73, 4885 (1993).

[13] O.K. Andersen, Phys. Rev. B 12, 3060 (1975).

[14] U. von Barth and L. Hedin, J. Phys. C 5, 1629 (1972).

[15] J. Friedel, Phil. Mag. 43, 153 (1952).

[16] Mathematica 2.2 (Wolfram Research, Inc. Champaign, IL, 1993).

[17] U. Klemradt, B. Drittler, R. Zeller and P.H. Dederichs, Phys. Rev. Lett. 64, 2803 (1990). 
[18] U. Klemradt, B. Drittler, T. Hoshino, R. Zeller, P.H. Dederichs and N. Stefanou, Phys. Rev. B 43, 9487 (1991).

[19] N.H. March, J. Nuc. Mater. 69 \& 70, 490 (1978) and references therein.

[20] T. Hoshino, R. Zeller and P.H. Dederichs, Phys. Rev. B 53, 8971 (1996).

[21] P.S. Ho and R. Benedek, IBM J. Res. Dev. 18, 386 (1974).

[22] N. Chetty, M. Weinert, T.S. Rahman and J.W. Davenport, Phys. Rev. B 52, 6313 (1995).

[23] R. W. Siegel, in Positron Annihilation, edited by P. G. Coleman, S. C. Sharma and L. M. Diana (North-Holland, Amsterdam, 1982), p. 351; K. Petersen, in Positron Solid-State Physics edited by W. Brandt and A. Dupasquier (North Holland, Amsterdam, 1983), p. 298.

[24] M.J. Puska and R. Nieminen, Rev. Mod. Phys. 66, 841 (1994).

[25] P.A. Sterne and J.H. Kaiser, Phys. Rev. B 43, 13892 (1991).

[26] B. Barbiellini, M.J. Puska, T. Korhonen, A. Harju, T. Torsti, and R.M. Nieminen, Phys. Rev. B 53, 16201 (1996).

[27] J. Arponen and E. Pajanne, Annals of Physics 121, 343 (1979).

[28] L. Lantto, Phys. Rev. B 36, 5160 (1987).

[29] M. Puska, J. Phys. Condens. Matter 3, 3455 (1991).

[30] W.D. Mosley, J.W. Dykes, P. Klavins, R.N. Shelton, P.A. Sterne, and R.H. Howell, Phys. Rev. B 48, 611 (1993).

[31] C. Colmenares, R.H. Howell, D. Ancheta, T. Cowan, J. Hanafee, and P. Sterne, UCID-2062296, Lawrence Livermore National Laboratory, p. 15 (1996).

[32] H. Ullmaier, Nuclear Fusion 24, 1039 (1984).

[33] L.K. Mansur, Journal of Nuclear Materials, 216, 97 (1994). 


\section{TABLES}

\begin{tabular}{|c|ccccccc|}
\hline Impurity & $\mathrm{Sc}$ & $\mathrm{Ti}$ & $\mathrm{Cu}$ & $\mathrm{Mg}$ & $\mathrm{Si}$ & $\mathrm{Ag}$ & $\mathrm{Au}$ \\
\hline$\Delta Q(e V)$ & 1.61 & - & 0.04 & -0.01 & -0.07 & -0.15 & -0.15 \\
\hline
\end{tabular}

Table 1: Experimental activation energies relative to $\mathrm{Al}, \Delta Q_{\text {exp }}$, for the diffusion of substitutional impurities in $\mathrm{Al}$ metal in the temperature range 600-900 $\mathrm{K}\left(Q_{\mathrm{Al}}=1.36 \mathrm{eV}\right)$. Positive values of $\Delta Q$ correspond to slow diffusers, i.e. slower than self-diffusion, and negative values correspond to fast diffusers.

\begin{tabular}{|c|ccccccc|}
\hline Impurity & $\mathrm{Sc}$ & $\mathrm{Ti}$ & $\mathrm{Cu}$ & $\mathrm{Mg}$ & $\mathrm{Si}$ & $\mathrm{Ag}$ & $\mathrm{Au}$ \\
\hline$B\left(a_{0}^{-1}\right)$ & 1.63 & 1.71 & 1.77 & 1.75 & 1.56 & 1.62 & 1.69 \\
$\Delta E_{21}(\mathrm{eV})$ & -0.19 & -0.28 & -0.18 & -0.04 & 0.02 & -0.08 & -0.02 \\
\hline
\end{tabular}

Table 2: Parameter $B$ obtained from fitting the total energies using equations (5) and (6). For comparison, the $\mathrm{Al}$ free-electron value of $B$ is $1.85 a_{0}^{-1}$. Also listed are the differences in energy between second-neighbour and nearest-neighbour impurity-vacancy configurations, $\Delta E_{21}=\varepsilon\left(R_{2}\right)-\varepsilon\left(R_{1}\right)$ as obtained from the fitted parameters. 


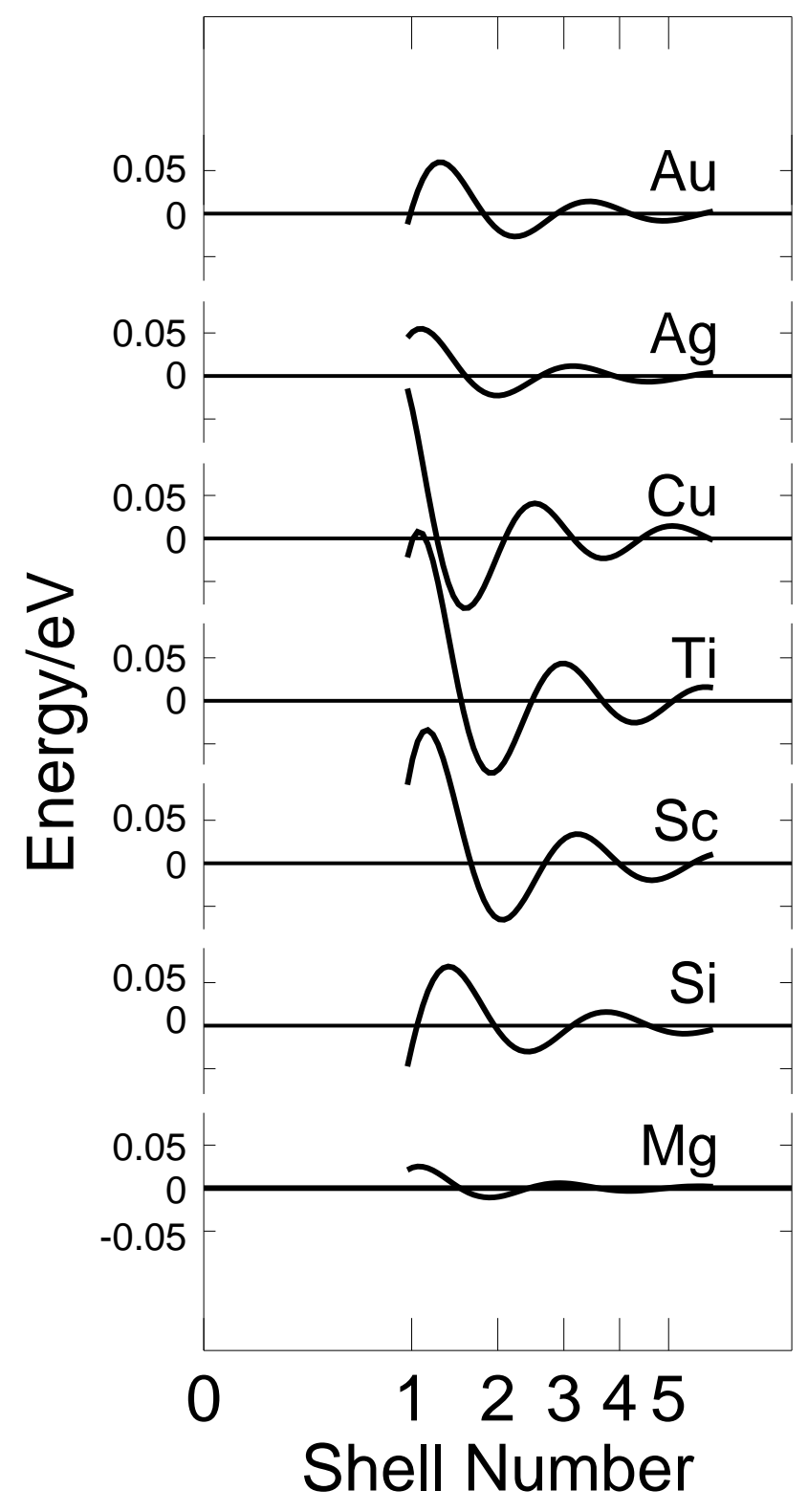

Figure 1: Impurity-vacancy interaction energy versus impurity-vacancy separation for various impurities in Al. Distances from the vacancy to the first through fifth shell of surrounding atoms in an fcc lattice are indicated. The curves represent the oscillating part of Eq. (5) while zero interaction energy corresponds to the Al-vacancy interaction in $\mathrm{Al}$ metal. 


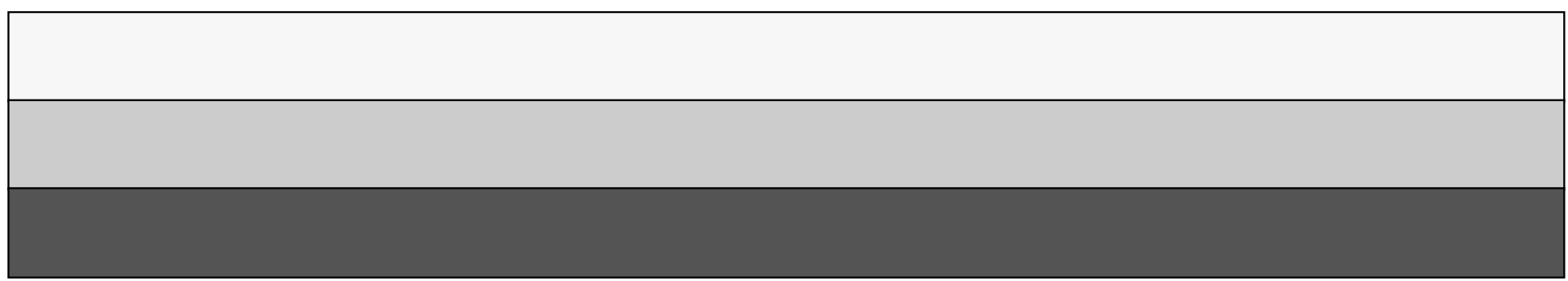

